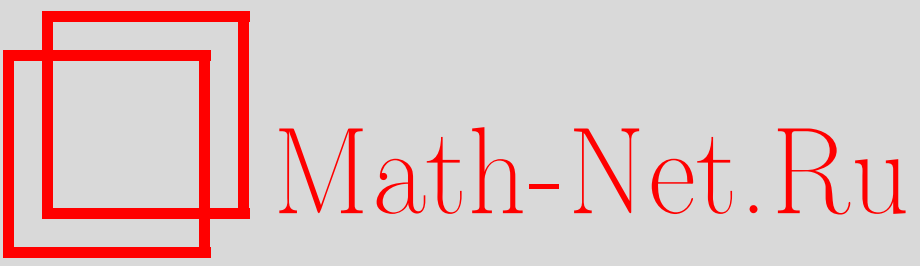

Н. Джоши, Регуляризация уравнения КдФ вблизи поверхности особенностей, TMФ, 2001, том 127, номер 3, 394-400

DOI: https://doi.org/10.4213/tmf467

Использование Общероссийского математического портала Math-Net.Ru подразумевает, что вы прочитали и согласны с пользовательским соглашением

http://www.mathnet.ru/rus/agreement

Параметры загрузки:

IP: 3.82 .47 .9

26 апреля 2023 г., 14:07:24 
ТЕОРЕТИЧЕСКАЯ

И МАТЕМАТИЧЕСКАЯ

ФИЗИКА

Том 127, № 3

июнь, 2001

(C) 2001 r.

Н. Джоши*

\section{РЕГУЛЯРИЗАЦИЯ УРАВНЕНИЯ КДФ ВБЛИЗИ ПОВЕРХНОСТИ ОСОБЕННОСТЕЙ}

Предложен метод регуляризации уравнения Кортевега-де Фриза вблизи, а не на поверхности особенностей. Более того, это позволяет нам показать, что для достаточно малых заданных при $x=0$ начальных условиях поверхность особенности существует поблизости и является аналитическим многообразием.

\section{1. ВВЕДЕНИЕ}

Сингулярности решений интегрируемых уравнений, подобных уравнению Кортевега-де Фриза (Кд $\Phi)$

$$
u_{x x x}=6 u u_{x}+u_{t},
$$

привлекают значительное внимание в последнее время. Интерес к ним связан с предположением Абловица-Рамани-Сегура (см. [1] и приведенную там литературу) о том, что все такие уравнения (точнее, их симметричные редукции) обладают свойством Пенлеве, т.е. все подвижные сингулярности решений являются полюсами. Однако несмотря на значительный интерес, многие фундаментальные вопросы, касающиеся глобальной структуры сингулярностей их решений, остаются открытыми.

В частности, не известно, все ли возможные сингулярные решения описываются рядами Пенлеве

$$
\sum_{j=0}^{\infty} u_{j}(x, t) \Phi^{j-\alpha}
$$

при целочисленном $\alpha$ (для уравнения $\mathrm{K} д \Phi \alpha=2$ ). Серьезным препятствием в решении этого вопроса являлось допушение, всегда делавшееся при исследовании сингулярностей, о том, что сингулярность появляется на заданной регулярной поверхности $\Phi=0$. В данной работе мы устраним это препятствие.

Рассмотрим задачу Коши для функций $u(x, t)$

$$
\left\{\begin{array}{l}
u_{x x x}=6 u u_{x}+u_{t} \\
\partial_{x}^{k} u(0, t)=\phi_{k}(t), \quad k=0,1,2
\end{array}\right.
$$

* Department of Pure Mathematics, University of Adelaide, Adelaide, SA 5005, Australia. E-mail: Nalini.Joshi@adelaide.edu.au 
где $x \in \Omega \subset \mathbb{C}$ и $0 \in \Omega$. Пусть заданы $t_{0} \in \mathbb{C}$ и $\rho_{0}>0$, а также положим, что $\Omega$ - замкнутый шар радиуса $\eta$, а функшия $\phi_{k}$ аналитична в замкнутом шаре $D_{\rho_{0}}\left(t_{0}\right)$ и велика в смысле, который уточняется ниже. Из теоремы Коши-Ковалевской следует сушествование единственного голоморфного решения в окрестности точки $\left(0, t_{0}\right)$, но о возможных особенностях решения ничего сказать нельзя. Мы покажем (при условии больших начальных условий), что сушествует голоморфное многообразие вблизи $x=0$, где решение имеет особенность.

Ряд авторов изучали особенности решений уравнения (1.1). Вайсс, Табор и Карневале [2] предложили процедуру формального разложения решений в ряд Лорана вблизи заданной регулярной нехарактеристической гиперповерхности. Киченассами и Литтман [3] предложили метод доказательства сходимости таких решений в виде рядов для нелинейных волновых уравнений и использовали его при исследовании особенностей уравнений, подобных уравнению $\mathrm{K} д \Phi$ в $\mathbb{R}^{2}[4]$. Джоши с соавторами обосновали альтернативное доказательство сходимости [5], оценили радиус сходимости в $\mathbb{C}^{2}$ и установили корректность задачи для уравнения КдФ с начальными значениями Вайсса-ТабораКарневале на заданной поверхности особенностей [6]. Ху и Ян [7] исследовали задачу сходимости разложений Пенлеве в терминах так называемых зеркальных систем.

Задачи с особенностями для нелинейных волновых уравнений хорошо известны. Для нелинейного волнового уравнения $\square u=\exp u$ с близкими к постоянным начальными условиями Киченассами [8] доказал сушествование поверхности особенностей и оценил ее регулярность. Каффарелли и Фридман [9] показали, что множество особенностей для уравнения $\square u=F(u)$ с суперлинейной неотрицательной функцией $F$ (при некотором условии на данные Коши) является регулярной гиперповерхностью. Однако подобного результата до сих пор не было получено для уравнения Кд $\Phi$.

Зафиксируем ветвь $\phi_{0}{ }^{1 / 2}$ и положим

$$
\begin{gathered}
v_{0}=\left(\frac{2}{\phi_{0}}\right)^{\frac{1}{2}}, \quad v_{1}=-\frac{v_{0}^{3} \phi_{1}}{4}, \quad v_{2}=\frac{1}{4 v_{0} v_{2}}\left(12 v_{1}^{2}-v_{0}^{4} \phi_{2}\right), \\
f(t):=\phi_{2}-3 \phi_{0}^{2}, \quad g(t):=\frac{\phi_{1}^{2}}{2}-\phi_{0}^{3}-\phi_{0} f(t), \\
\tilde{f}(t):=f(t)-2 \frac{v_{0}^{\prime}(t)}{v_{0}^{2}}, \quad \tilde{g}(t):=g(t)+2 \frac{v_{0}^{\prime}(t)}{v_{0}^{4}}+\frac{1}{4}\left(\ln v_{0}\right)^{\prime \prime},
\end{gathered}
$$

где штрих обозначает дифференцирование по $t$. Следуюшие условия на начальные данные необходимы для получения нашего результата.

ОПРЕДЕЛЕНИЕ. Для заданного достаточно малого $\epsilon_{0}<1 / 2$ начальные условия называются допустимыми , если при всех $0<\epsilon<\epsilon_{0}$ выполнены неравенства

$$
\left\|v_{0}\right\|_{\rho} \leqslant \epsilon, \quad\left\|v_{1}-1\right\|_{\rho} \leqslant \epsilon, \quad\left\|v_{2}\right\|_{\rho} \leqslant 2 \epsilon
$$

и

$$
\|\tilde{f}\|_{\rho}<\frac{1}{2 \epsilon^{2}}, \quad\|\tilde{g}\|_{\rho}<\frac{1}{\epsilon}
$$

где $\|\cdot\|_{\rho}$ обозначает sup-норму на $D_{\rho}\left(t_{0}\right)$.

Нашим основным результатом является 
ТЕОРема. Можно так выбрать $0<\rho \leqslant \rho_{0}$ и аналитическую на $D_{\rho}\left(t_{0}\right)$ функиию $\vartheta(t)$, что задача с начальными условиями (1.2) при допустимых начальных данных имеет единственное решение в области

$$
\left\{(x, t)\left|t \in D_{\rho}\left(t_{0}\right), 0 \leqslant\right| x|<| \vartheta(t) \mid\right\}
$$

такое, что $|u(x, t)| \rightarrow \infty$ при $x \rightarrow \vartheta(t) \quad \forall t \in D_{\rho}\left(t_{0}\right)$.

Мы докажем эту теорему в другой публикации, здесь же представим основной метод регуляризации, приводящий к этому результату.

\section{2. РЕГУЛЯРИЗАЦИЯ УРАВНЕНИЯ КДФ}

Первый шаг доказательства теоремы состоит в замене задачи Коши (1.2) на подходящее интегральное уравнение. Определим функцию

$$
u(x, t):=\frac{2}{v(x, t)^{2}}
$$

Всякий квадратный корень, возникающий при обрашении этих соотношений, предполагается фиксированным с помощью сделанного выбора для $v_{0}$. Кроме того, мы полагаем, что $t_{0}=0$ (после сдвига, если требуется), а также что $v_{0}^{\prime}$ и $v_{0}^{\prime \prime}$ ограничены некоторым положительным числом $b$.

Вспоминая, что решение велико вблизи $x=0$, мы дважды последовательно проинтегрируем уравнение КдФ таким образом, как если бы в нем присутствовали только доминантные члены $u_{x x x}$ и $u u_{x}$ :

$$
\begin{aligned}
& u_{x x}=3 u^{2}+\int_{0}^{x} u_{t}(\xi, t) d \xi+f(t) \\
& \frac{u_{x}^{2}}{2}=u^{3}+I+f(t) u+g(t)
\end{aligned}
$$

где при получении второго интегрального уравнения используется интегрирующий множитель $u_{x}$ и

$$
I:=\int_{0}^{x} \int_{0}^{\eta} u_{x}(\eta, t) u_{t}(\xi, t) d \xi d \eta .
$$

При подстановке (2.1) уравнение КдФ и полученные в результате его интегрирования уравнения $(2.2 \mathrm{a}),(2.2 б)$ преобразуются к виду

$$
\begin{aligned}
v_{x x x} & =9 \frac{v_{x} v_{x x}}{v}-12 v_{x} \frac{v_{x}^{2}-1}{v^{2}}+v_{t}, \\
v_{x x} & =3 \frac{v_{x}^{2}-1}{v}-\frac{v^{3}}{2} \partial_{t}\left(\int_{0}^{x} \frac{d x}{v^{2}}\right)-\frac{f(t)}{4} v^{3}, \\
v_{x}^{2} & =1+\frac{v^{6}}{8} I+\frac{f(t)}{4} v^{4}+\frac{g(t)}{8} v^{6} .
\end{aligned}
$$


Эти уравнения представляются сингулярными, поскольку произвольно малый член $v$ входит в знаменатель нескольких слагаемых. Эта кажушаяся сингулярность появляется также и в члене $I$, который преобразуется (после изменения порядка интегралов и явного интегрирования) в

$$
I=\frac{4}{v(x, t)^{2}} \partial_{t} \int_{0}^{x} \frac{d \eta}{v(\eta, t)^{2}}-2 \partial_{t} \int_{0}^{x} \frac{d \eta}{v(\eta, t)^{4}} .
$$

Тем не менее в следуюшей лемме мы покажем, что преобразованная система в действительности регулярна.

ЛЕмма 1. 1. Уравнение КдФ әквивалентно системе

$$
\begin{aligned}
& v_{x}=p \\
& p_{x}=3 v A-\frac{v q}{2}+v^{3} B \\
& q_{x}=p_{t} \\
& r_{x}=15 v p A-\frac{9}{2} p q+q+9 v^{2} p B \\
& s_{x}=r_{t} \\
& \tau_{x}=s_{t}, \\
& y_{x}=z_{t}, \\
& z_{x}=-2 \frac{p q}{p+1} A+\frac{3}{2} p^{2} w+10 p^{2} B+12 A^{2}-5 A q+ \\
& \quad+\frac{q^{2}}{2}+10 A B-2 v^{2} A B+2 v^{4} B^{2}, \\
& w_{x}=\frac{q s}{2}-\frac{p \tau}{2}-\frac{v s_{t}}{4}-4 \partial_{t}\left(\frac{A}{p+1}\right)+2 v p C+ \\
& \quad+v^{2} \frac{1}{4} \partial_{t}\left(\frac{w(p+1)+p q A+v y(p+1)-v s A}{(p+1)^{2}}\right),
\end{aligned}
$$

$2 \partial e$

$$
\begin{aligned}
A & :=\frac{q}{4}+\frac{v^{2}}{8} w \\
B & :=\frac{1}{2} \partial_{t}\left(\int_{0}^{x} \frac{A}{p+1}\right)-\frac{\tilde{f}}{4} \\
C & :=\tilde{g}+\frac{1}{4} \partial_{t}\left(\int_{0}^{x}\left[\frac{w+q A+v A_{t}}{p+1}-\frac{(q+v s) A}{(p+1)^{2}}\right]\right) .
\end{aligned}
$$

2. В частности,

$$
\begin{gathered}
v_{x}^{2}=1+v^{2} A(x, t) \\
w(x, t)=2 \tilde{f}(t)+\tilde{g}(t) v^{2}+\frac{q^{2}}{4}-\frac{v \tau}{4}-4 \partial_{t} \int_{0}^{x} d x \frac{A}{p+1}+ \\
+\frac{v^{2}}{4} \partial_{t} \int_{0}^{x} d x\left(\frac{w}{p+1}-\frac{q A}{(p+1)^{2}}+\partial_{t}\left(\frac{v A}{p+1}\right)\right) .
\end{gathered}
$$


ДокАЗАтЕльство. Рассмотрим уравнение (2.4в). Для того чтобы сделать возможным явное интегрирование первого члена в $I$, перепишем

$$
\frac{1}{v^{2}}=\frac{v_{x}}{v^{2}}-\frac{v_{x}^{2}-1}{v^{2}\left(v_{x}+1\right)}
$$

и воспользуемся уравнением $(2.4 \mathrm{~B})$ для замены $v_{x}^{2}-1$, что дает

$$
\int_{0}^{x} \frac{d \eta}{v(\eta, t)^{2}}=-\frac{1}{v(x, t)}+\frac{1}{v_{0}(t)}-\int_{0}^{x} \frac{d \eta}{v_{x}+1}\left(\frac{v^{4}}{8} I+\frac{f(t)}{4} v^{2}+\frac{g(t)}{8} v^{4}\right)
$$

Аналогично имеем

$$
\int_{0}^{x} \frac{d \eta}{v(\eta, t)^{4}}=-\frac{1}{3 v(x, t)^{3}}+\frac{1}{3 v_{0}(t)^{3}}-\int_{0}^{x} \frac{d \eta}{v_{x}+1}\left(\frac{v^{2}}{8} I+\frac{f(t)}{4}+\frac{g(t)}{8} v^{2}\right) .
$$

Это подсказывает замену переменных $I=: 2\left(v_{t} / v^{4}\right)+J$. Тогда уравнение (2.4в) принимает вид

$$
v_{x}^{2}=1+\frac{v^{2} v_{t}}{4}+\frac{v^{6}}{8} J+\frac{f(t)}{4} v^{4}+\frac{g(t)}{8} v^{6},
$$

где

$$
\begin{aligned}
J= & \frac{4}{v^{2}}\left\{\left(\frac{1}{v_{0}(t)}\right)_{t}-\partial_{t} \int_{0}^{x} \frac{d \eta}{v_{x}+1}\left(\frac{v_{t}}{4}+\frac{v^{4}}{8} J+\frac{f(t)}{4} v^{2}+\frac{g(t)}{8} v^{4}\right)\right\}+ \\
& +2\left\{-\left(\frac{1}{3 v_{0}(t)^{3}}\right)_{t}+\partial_{t} \int_{0}^{x} \frac{d \eta}{v_{x}+1}\left(\frac{v_{t}}{4 v^{2}}+\frac{v^{2}}{8} J+\frac{f(t)}{4}+\frac{g(t)}{8} v^{2}\right)\right\}
\end{aligned}
$$

Рассмотрим теперь кажущийся сингулярным первый член в последнем интеграле. Преобразуем его:

$$
\begin{aligned}
\frac{v_{t}}{2 v^{2}\left(v_{x}+1\right)} & =\frac{v_{t}}{2 v^{2}}\left(\frac{1}{2}-\frac{v_{x}^{2}-1}{2\left(v_{x}+1\right)^{2}}\right)= \\
& =-\left(\frac{1}{4 v}\right)_{t}-\frac{v_{t}}{4\left(v_{x}+1\right)^{2}}\left(\frac{v_{t}}{4}+\frac{v^{4}}{8} J+\frac{f(t)}{4} v^{2}+\frac{g(t)}{8} v^{4}\right)
\end{aligned}
$$

Аналогично все еще потенциально сингулярный первый член в правой части получившегося выражения дает

$$
\begin{aligned}
-\frac{1}{4} \partial_{t t} \int_{0}^{x} \frac{d \eta}{v(\eta, t)}= & -\frac{1}{4} \partial_{t t}\left(\ln \frac{v}{v_{0}}\right)+ \\
& +\frac{1}{4} \partial_{t t} \int_{0}^{x} \frac{d \eta}{v_{x}+1}\left(\frac{v v_{t}}{4}+\frac{v^{5}}{8} J+\frac{f(t)}{4} v^{3}+\frac{g(t)}{8} v^{5}\right)
\end{aligned}
$$

Переписьвая

$$
J=: \frac{K}{v^{2}}+\frac{4}{v^{2}}\left(\frac{1}{v_{0}(t)}\right)_{t}-2\left(\frac{1}{3 v_{0}(t)^{3}}\right)_{t}+\frac{1}{4} \partial_{t t}\left(\ln v_{0}\right)
$$


из соотношения (2.9) получаем

$$
\begin{aligned}
K(x, t)= & \frac{v_{t}^{2}}{4}-\frac{v v_{t t}}{4}-4 \partial_{t} \int_{0}^{x} \frac{d \xi}{v_{x}(\xi, t)+1}\left(\frac{v_{t}}{4}+\frac{v^{2} K}{8}+\frac{v^{2} \tilde{f}(t)}{4}+\frac{v^{4} \tilde{g}(t)}{8}\right)+ \\
& +v^{2} \partial_{t}\left\{2 \int_{0}^{x} \frac{d \xi}{v_{x}(\xi, t)+1}\left(\frac{K}{8}+\frac{\tilde{f}(t)}{4}+\frac{v^{2} \tilde{g}(t)}{8}\right)-\right. \\
& -\frac{1}{4} \int_{0}^{x} \frac{d \xi v_{t}}{\left(v_{x}(\xi, t)+1\right)^{2}}\left(\frac{v_{t}}{4}+\frac{v^{2} K}{8}+\frac{v^{2} \tilde{f}(t)}{4}+\frac{v^{4} \tilde{g}(t)}{8}\right)+ \\
& \left.+\frac{\partial_{t}}{4} \int_{0}^{x} \frac{d \xi v}{v_{x}(\xi, t)+1}\left(\frac{v_{t}}{4}+\frac{v^{2} K}{8}+\frac{v^{2} \tilde{f}(t)}{4}+\frac{v^{4} \tilde{g}(t)}{8}\right)\right\} .
\end{aligned}
$$

Записывая

$$
w:=K+2 \tilde{f}(t)+v^{2} \tilde{g}(t)
$$

приходим к результатам (2.7) и (2.8), где $A$ определяется формулой (2.6а) и производные $v$ обозначены как

$$
p=v_{x}, \quad q=v_{t}, \quad r=v_{x x}, \quad s=v_{x t}, \quad \tau=v_{t t}, \quad y=A_{t}, \quad z=A_{x} .
$$

Тем самым доказано утверждение 2 леммы.

Обозначения (2.10) вместе с определениями (2.6) немедленно приводят и к уравнениям $(2.5 \mathrm{a}),(2.5 \mathrm{~B})$ и $(2.5$ д)-(2.5ж).

Чтобы получить (2.5б), используем уравнения (2.4б) и (2.7) и проинтегрируем аналогично предыдушему. Чтобы получить (2.5г), подставим (2.7) и (2.5б) в уравнение (2.4a). Уравнение (2.5и) получается дифференцированием (2.8). Чтобы получить (2.53), дважды продифференцируем $A=\left(v_{x}^{2}-1\right) / v^{2}$ и воспользуемся уравнениями (2.4a) и (2.5б). Это завершает доказательство леммы.

Полагая $v=: \epsilon \theta$ и $x=: \epsilon \xi$, определим

$$
\begin{gathered}
w_{0}:=\frac{4}{v_{0}^{4}}\left(-2 v_{0}^{2} v_{0}^{\prime}+v_{1}^{2}-1\right), \quad A_{0}:=\frac{1}{8}\left(2 v_{0}^{\prime}+v_{0}^{2} w_{0}\right), \\
y_{0}:=A_{0}^{\prime}, \quad z_{0}:=\frac{2}{v_{0}^{2}}\left(v_{1} v_{2}-2 v_{1} v_{0} A_{0}\right)^{\prime}, \\
\left.\qquad \begin{array}{c}
\theta-v_{0} / \epsilon \\
p-v_{1} \\
q-v_{0}^{\prime} \\
r-v_{2} \\
s-v_{1}^{\prime} \\
\tau-v_{0}^{\prime \prime} \\
y-y_{0} \\
z-z_{0} \\
w-w_{0}
\end{array}\right) .
\end{gathered}
$$


Перепишем систему уравнений (2.5) как

$$
\mathrm{V}_{\xi}=\mathcal{F}(\mathrm{V}, \xi)
$$

Пусть задано достаточно малое $\epsilon_{0}$, причем $0<\epsilon<\epsilon_{0}$. Пусть $\mathcal{S}=\left\{B_{\rho}\right\}_{0<\rho<\rho_{0}}$ - масштаб банаховых пространств аналитических функций на вложенных замкнутых дисках $D_{\rho}(0)$. Далее мы полагаем, что $0<\mu<\epsilon, 0<\eta<1 / 2$, а также считаем $\rho$ и $\rho^{\prime}$ такими, что $0 \leqslant \rho^{\prime}<\rho<\rho_{0}$.

Лемма 2. Отображсение $(\mathrm{V}, \xi) \mapsto \mathcal{F}(\mathrm{V}, \xi)$ :

$$
\left\{\mathrm{V} \in B_{\rho}:\|\mathrm{V}\|_{\rho}<\mu\right\} \times\{\xi:|\xi|<\eta\} \stackrel{\mathcal{F}}{\rightarrow} B_{\rho^{\prime}}
$$

является аналитическим.

ДокАЗАТЕльСтво. Для доказательства требуется только показать, что $p+1$ не может обращаться в нуль. Это верно, поскольку

$$
\left|p-p_{0}\right|<\epsilon \Leftrightarrow\left|p-1-\left(v_{1}-1\right)\right|<\epsilon \Rightarrow|p-1|-\left|v_{1}-1\right|<\epsilon \Rightarrow|p-1|<2 \epsilon .
$$

Отсюда получаем

$$
|p+1|=|2+p-1|>2-2 \epsilon>1 \text {. }
$$

Лемма доказана.

Результаты, подобные приведенному, позволяют показать, что уравнение (2.11) имеет аналитическое решение, и более того, сушествует нуль этого решения $v(x, t)$ вблизи $x=0$, откуда и вытекает приведенная в разделе 1 теорема.

Благодарности. Работа поддержана Australian Research Council (грант № $\mathrm{A} 69803721$ и стипендия № F69700172).

\section{Список литературы}

[1] M. J. Ablowitz, P. A. Clarkson. Solitons, Nonlinear Evolution Equations, and Inverse Scattering. London Math. Soc. Lect. Notes Math. V. 149. Cambridge: Cambridge Univ. Press, 1991.

[2] J. Weiss, M. Tabor, G. Carnevale. J. Math. Phys. 1983. V. 24. P. 522-526.

[3] S. Kichenassamy, W. Littman. Commun. Part. Diff. Equat. 1993. V. 18. P. 431-452.

[4] S. Kichenassamy, W. Littman. Commun. Part. Diff. Equat. 1993. V. 18. P. 1869-1899.

[5] N. Joshi, J. A. Petersen. Nonlinearity. 1994. V. 7. P. 595-602.

[6] N. Joshi, G. K. Srinivasan. Nonlinearity. 1997. V. 10. P. 71-79.

[7] J. Hu, M. Yan. Stud. Appl. Math. 2000. V. 104. P. 67-90.

[8] S. Kichenassamy. Commun. Part. Diff. Equat. 1996. V. 21. P. 125-162.

[9] L. Caffarelli, A. Friedman. Trans. Am. Math. Soc. 1986. V. 297. P. 223-241. 